\title{
A New Basic Unit for Symmetric and Asymmetric Cascaded Multilevel Inverters with Reduced Power Electronic Devices
}

\author{
Maryam Sarbanzadeh ${ }^{1}$, Student Member, IEEE, Mohammad Ali Hosseinzadeh ${ }^{1}$, Student Member, IEEE, \\ Ebrahim Babaei ${ }^{2}$, Member, IEEE, Marco Rivera ${ }^{1}$, Member, IEEE \\ ${ }^{1}$ Faculty of Engineering, University of Talca, Talca, Chile \\ ${ }^{2}$ Faculty of Electrical and Computer Engineering, University of Tabriz, Tabriz, Iran \\ E-mails:maryam_sarbanzadeh@yahoo.com; m.a_hosseinzadeh@yahoo.com; e-babaei@tabrizu.ac.ir;mrivera@utalca.cl
}

\begin{abstract}
In this paper, a new basic unit for the cascaded multilevel inverter is proposed. By series-connected numbers of the proposed basic unit, a new cascaded multilevel inverter is also proposed. In order to generate all (even and odd) voltage levels two new algorithms to determine the magnitude of dc voltage sources are proposed. Then, this inverter is compared with several conventional cascaded multilevel inverters that have been presented in the literature. Based on these comparisons, the proposed inverter is able to generate a higher number of output levels by using a lower number of power electronic devices, dc voltage sources and minimum variety of the value of dc voltage sources. As a result, high efficiency, reducing the size and cost of the inverter is the other advantages of the proposed inverter. Finally, the accuracy performance of the proposed cascaded inverter to generate all voltage levels is verified through experimental results on a 49-level inverter in a laboratory prototype.
\end{abstract}

Keywords- Basic unit; multilevel inverters; cascaded multilevel inverter; power electronic devices

\section{INTRODUCTION}

A multilevel inverter is a power electronic converter that generates desired ac output levels by using several dc voltage sources as inputs [1]. By increasing the number of dc voltage sources and semiconductor switches a sinusoidal-like waveform is generated at the output. Recently multilevel inverters have received more attention because of their capability in high power, high voltage applications. In addition, high power quality, reducing lower order harmonics, lower switching losses and better electromagnetic interference are other advantages of multilevel inverters [2], [3]. There are three main categories for multilevel inverters: Diode clamped multilevel inverter [4], flying capacitor multilevel inverter [5] and cascaded multilevel inverter [6]. The cascaded multilevel inverter is divided into two groups: symmetric cascaded multilevel inverter and asymmetric cascade multilevel inverter. In the symmetric state, the magnitudes of all dc voltage sources are the same while in the asymmetric state the magnitudes of dc voltage sources are different [7], [8]. In the cascaded multilevel inverter, there are any many diodes clamped and flying capacitor. In addition, reducing the stress on power switches and faulttolerant operations, extendibility, modularization, simplicity of control and reliability are some of the main advantages of these inverters in comparisons to two other multilevel inverters. However, a high number of required dc voltage sources and semiconductor power switches by increasing the number of generated output levels are disadvantageous of this inverter [9], [10].

The first cascaded multilevel inverter has been presented in [11]. This inverter is comprised of series connected of several H-bridges. In order to increase the number of output levels, different algorithms to determine the magnitudes of the dc voltage sources in the $\mathrm{H}$-bridge have been presented in [11], [12]. Then, two other symmetric cascaded multilevel inverters have been presented in [13], [14]. The main problems of the symmetric cascaded inverters is a high number of required integrated gate bipolar transistors (IGBTs), dc voltage sources, power switches, and driver circuits by increasing the number of generated output levels because of its low magnitude of dc voltage sources. In [1517], three other cascaded multilevel inverters have been presented. Three different algorithms to determine the magnitude of $\mathrm{dc}$ voltage sources as symmetric and asymmetric states have been presented for the presented inverter in [15]. In addition, two different algorithms have been considered for each of the presented inverters in [16] and [17].

Although all of other topologies have their own advantages in this paper, in order to increase the number of output levels by using a lower number of power electronic devices and $\mathrm{dc}$ voltage sources, a new basic unit is proposed. Then, by the series connection of the numbers of these basic units, a new cascaded multilevel inverter is introduced. In order to generate all positive and negative levels at the output two algorithms to determine the magnitude of dc voltage sources are proposed. The proposed inverter is compared with several conventional cascaded multilevel inverters to investigate its advantages and disadvantages. Finally, the accuracy performance of the proposed inverters is reconfirmed by using experimental results on a 49-level proposed inverter.

\section{PROPOSED BASIC UNIT}

The proposed basic unit is shown in Fig. 1. As shown in this figure, the unidirectional and bidirectional power switches are used in this topology. Each unidirectional power switch consists of an IGBT with an anti-parallel diode that is able to conduct current in both direction and block voltage in one polarity. While the bidirectional power switch includes two 
IGBTs with Two anti-parallel diodes that conduct current in both directions and blocks voltage in two positive and negative polarities. As a result, the unidirectional switch requires one driver circuit and bidirectional switch needs two driver circuits unless a common emitter structure is used. In this condition, each bidirectional power switch needs one driver circuit. In this topology, the power switches of $T_{1}$, $T_{2}, T_{3}, S_{1}$ and $S_{m}$ are unidirectional switches while the power switches of $S_{2}, S_{3}, \ldots, S_{m-2}, S_{m-1}$ and $T_{3}$ are bidirectional power switches.

In addition, in order to generate the same steps output levels, the magnitudes of dc voltage sources have to be considered equally. In this topology, the power switches of $T_{1}$ is used to generate positive levels, the switch $T_{2}$ is used to generate negative levels and $T_{3}$ is used to generate zero levels. Table I shows the switching pattern and generated output levels in the proposed basic unit. As it is obvious from this Table, the proposed topology is able to generate positive and negative voltage levels at the output. Moreover, in order to generate all voltage levels at the output except the zero levels, only two power switches in different operating modes are turned on. In order to simplify, the voltage drop of switch conduction state is eliminated.

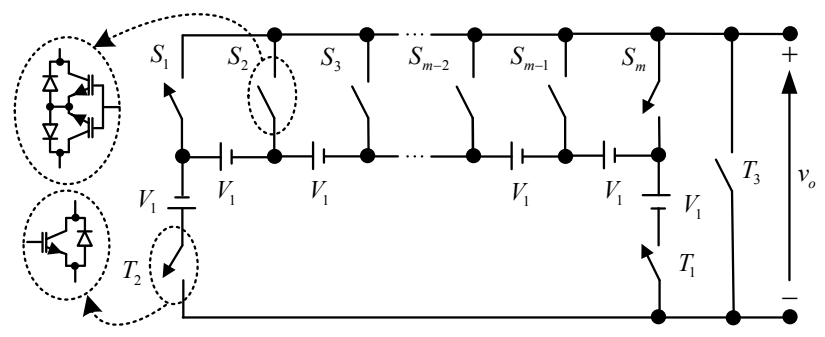

Fig. 1: The proposed basic unit

TABLE I. THE GENERATED UOTPUT LEVELS IN DIFFERENT SWITCHING PATTERN

\begin{tabular}{|c|c|c|c|c|c|c|c|c|c|c|}
\hline$v_{o}$ & $S_{1}$ & $S_{2}$ & $S_{3}$ & $\cdots$ & $S_{m-2}$ & $S_{m-1}$ & $S_{m}$ & $T_{1}$ & $T_{2}$ & $T_{3}$ \\
\hline$m V_{1}$ & 1 & 0 & 0 & $\cdots$ & 0 & 0 & 0 & 1 & 0 & 0 \\
\hline$(m-1) V_{1}$ & 0 & 1 & 0 & $\cdots$ & 0 & 0 & 0 & 1 & 0 & 0 \\
\hline$(m-2) V_{1}$ & 0 & 0 & 1 & $\cdots$ & 0 & 0 & 0 & 1 & 0 & 0 \\
\hline$\vdots$ & $\vdots$ & $\vdots$ & $\vdots$ & $\cdots$ & $\vdots$ & $\vdots$ & $\vdots$ & $\vdots$ & $\vdots$ & $\vdots$ \\
\hline $3 V_{1}$ & 0 & 0 & 0 & 0 & 1 & 0 & 0 & 1 & 0 & 0 \\
\hline $2 V_{1}$ & 0 & 0 & 0 & $\cdots$ & 0 & 1 & 0 & 1 & 0 & 0 \\
\hline$V_{1}$ & 0 & 0 & 0 & $\cdots$ & 0 & 0 & 1 & 1 & 0 & 0 \\
\hline 0 & 0 & 0 & 0 & $\cdots$ & 0 & 0 & 0 & 0 & 0 & 1 \\
\hline$-V_{1}$ & 1 & 0 & 0 & $\cdots$ & 0 & 0 & 0 & 0 & 1 & 0 \\
\hline$-2 V_{1}$ & 0 & 1 & 0 & $\cdots$ & 0 & 0 & 0 & 0 & 1 & 0 \\
\hline$-3 V_{1}$ & 0 & 0 & 1 & $\cdots$ & 0 & 0 & 0 & 0 & 1 & 0 \\
\hline$\vdots$ & $\vdots$ & $\vdots$ & $\vdots$ & $\cdots$ & $\vdots$ & $\vdots$ & $\vdots$ & $\vdots$ & $\vdots$ & $\vdots$ \\
\hline$-(m-2) V_{1}$ & 0 & 0 & 0 & $\cdots$ & 1 & 0 & 0 & 0 & 1 & 0 \\
\hline$-(m-1) V_{1}$ & 0 & 0 & 0 & $\cdots$ & 0 & 1 & 0 & 0 & 1 & 0 \\
\hline$-m V_{1}$ & 0 & 0 & 0 & $\cdots$ & 0 & 0 & 1 & 0 & 1 & 0 \\
\hline
\end{tabular}

In the proposed basic unit, the number of generated output levels $\left(N_{\text {level,unit }}\right)$, power switches $\left(N_{\text {switch,unit }}\right)$, IGBTs $\left(N_{\text {IGBT,unit }}\right)$, driver circuits $\left(N_{\text {driver,unit }}\right)$, dc voltage sources $\left(N_{\text {source,unit }}\right)$ and the maximum output voltage levels $\left(V_{o, \text { max }, \text { unit }}\right)$ are calculated as follows, respectively:

$$
\begin{aligned}
& N_{\text {level,unit }}=2 m+1 \\
& N_{\text {switch,unit }}=m+3 \\
& N_{\text {IGBT,unit }}=2 m+2 \\
& N_{\text {driver,unit }}=m+3 \\
& N_{\text {source,unit }}=m+1 \\
& V_{o, \text { max ,unit }}=m V_{1}
\end{aligned}
$$

In the above equations $m$ is the number of used power switches in the proposed basic unit without considering the constant power switches $T_{1}, T_{2}, T_{3}$.

One of the main parameters to determine the cost of the multilevel inverters is the value of power switches which is completely depended on the voltage and current of the power switch. Therefore, by reducing the current and voltage values of power switches the cost of the inverter is decreased [12].

Generally, in multilevel inverters, the current value of each power switch is determined by the load current value while the blocked voltage values of the power switches are different. As a result, the maximum blocked voltage value of power switches has the most important influence in the cost of the inverter. The value of this parameter is depending on the states of the switches.

According to Fig. 1, the maximum value of the blocked voltage by unidirectional power switches $T_{1}, T_{2}, T_{3}$ are obtained as follow:

$V_{T 1}=V_{T 2}=(m+1) V_{1}$

$V_{T 3}=m V_{1}$

The blocked voltage by unidirectional switches of $S_{1}$ and $S_{m}$ are the same and obtained as follows:

$V_{S 1}=V_{S m}=(m-1) V_{1}$

The maximum blocked voltage by other bidirectional power switches are calculated as follows:

$$
\begin{aligned}
& V_{S 2}=V_{S(m-1)}=(m-2) V_{1} \\
& V_{S 3}=V_{S(m-2)}=(m-3) V_{1} \\
& \vdots \\
& V_{S\left(\frac{m}{2}\right)}=V_{S\left(\frac{m+2}{2}\right)}=\left(m-\left(\frac{m}{2}\right)\right) V_{1} \\
& \quad=\left(\frac{m}{2}\right) \quad \text { if m:even }
\end{aligned}
$$




$$
\begin{aligned}
V_{S\left(\frac{m-1}{2}\right)} & =V_{S\left(\frac{m+3}{2}\right)}=\left(m-\left(\frac{m-1}{2}\right)\right) V_{1} \\
& =\left(\frac{m}{2}+\frac{1}{2}\right) \quad \text { if } m: \text { odd } \\
V_{S\left(\frac{m+1}{2}\right)} & =\left(m-\left(\frac{m+1}{2}\right)\right) V_{1} \\
& =\left(\frac{m}{2}-\frac{1}{2}\right) \quad \text { if } m: \text { odd }
\end{aligned}
$$

Therefore, the blocked voltage by used power switches $\left(V_{\text {block,unit }}\right)$ in the proposed basic unit is obtained as follows:

$$
\begin{aligned}
V_{\text {block, unit }} & =V_{T 1}+V_{T 2}+V_{T 3}+V_{S 1}+V_{S m}+V_{S 2}+V_{S 3}+\cdots \\
& =\left(\frac{3}{4} m^{2}+\frac{5}{2} m+2\right) V_{1} \text { if } m: \text { even } \\
& =\left(\frac{3}{4} m^{2}+\frac{5}{2} m+\frac{7}{4}\right) V_{1} \text { if } m: \text { odd }
\end{aligned}
$$

\section{SUGGESTED CASCADED MULTILEVELINVERTER}

It is possible to series connected $n$ numbers of the proposed basic unit to generate a new cascaded multilevel inverter. Fig. 2 shows the proposed cascaded multilevel inverter. For this topology the number of power switches $\left(N_{\text {switch }}\right)$, IGBTs $\left(N_{\text {IGBT }}\right)$, driver circuits $\left(N_{\text {driver }}\right)$, dc voltage sources $\left(N_{\text {source }}\right)$ and the amount of blocked voltage by power switches $\left(V_{\text {block,total }}\right)$ are calculated as follows, respectively:

$$
\begin{aligned}
& N_{\text {switch }}=\sum_{i=1}^{n}\left(m_{i}+3\right) \\
& N_{\text {IGBT }}=\sum_{i=1}^{n}\left(2 m_{i}+2\right) \\
& N_{\text {driver }}=\sum_{i=1}^{n}\left(m_{i}+3\right) \\
& N_{\text {source }}=\sum_{i=1}^{n}\left(m_{i}+1\right)
\end{aligned}
$$

It is possible to develop the proposed inverter in both single and three phase system algorithms to determine the magnitude of dc voltage sources. So we propose two different methods to determine of dc sources magnitudes.

\section{A. First Proposed Method}

In the first proposed method, the magnitudes of all dc voltage sources are considered equally. In other word:

$$
V_{1}=V_{2}=\cdots \quad V_{\mathrm{dc}}
$$

The proposed inverter based on this method is known as symmetric cascaded multilevel inverter. In this condition, the number of generated output level is obtained as follows:

$N_{\text {level }}=2 n m+1$

\section{B. Second Proposed Method}

In the second proposed method the magnitudes of dc voltage sources are considered as follows:

First unit:

$V_{1}=V_{d c}$

\section{Second unit:}

$V_{2}=\left(2 m_{1}+1\right) V_{d c}$

Third unit:

$$
\begin{aligned}
& V_{3}=\left(2 m_{1}+1\right)\left(2 m_{2}+1\right) V_{d c} \\
& n^{\text {th }} \text { unit: } \\
& V_{n}=2 \sum_{i=1}^{n-1} m_{i} V_{i}+V_{d c} \\
& \left.=\left[\left(2 m_{1}+1\right)\left(2 m_{2}+1\right)\left(2 m_{3}+1\right) \cdots+1\right)\right] V_{d c}
\end{aligned}
$$

By considering $m_{1}=m_{2}=\cdots \quad \ldots \quad m$, the number of levels from (22) and (24) are recalculated as follows:

$$
N_{\text {level }}=(2 m+1)^{n}
$$

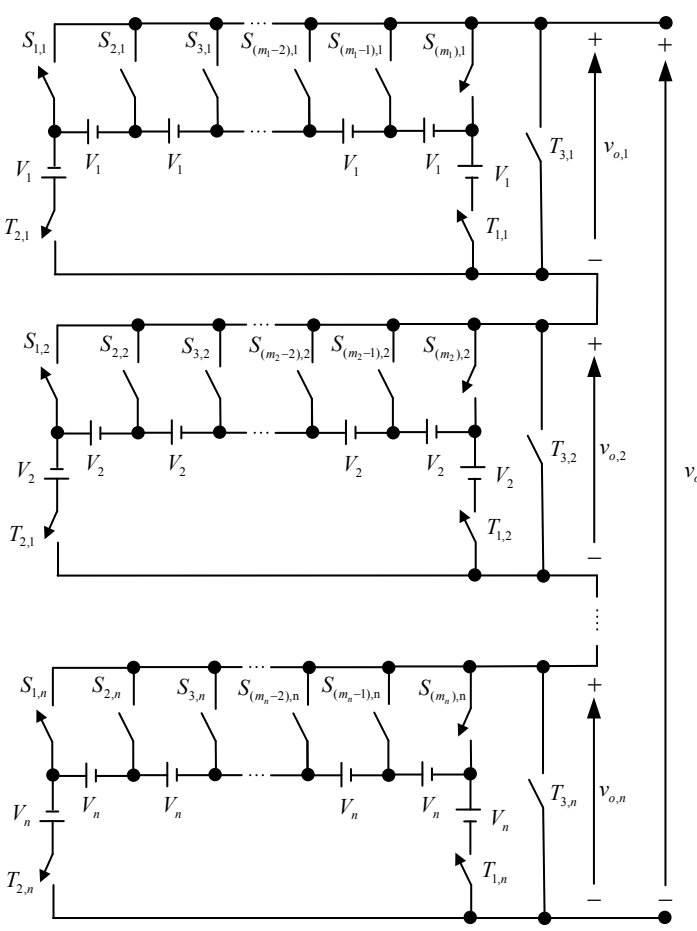

Fig. 2. The proposed cascaded multilevel inverter 


\section{COMPARISON OF THE PROPOSED CASCADED INVERTERS WITH MULTILEVEL INVERTERS}

In this section, the proposed cascaded multilevel inverter is compared with several cascaded multilevel inverters that have been presented in literature. This comparison is from the number of IGBTs, power switches, and dc voltage sources. In this comparison, the proposed cascaded multilevel inverter based on its algorithms are shown $P_{1}, P_{2}$. The H-bridge cascaded multilevel inverter with three symmetric and asymmetric algorithms that have been presented in [11], [12] are indicated $R_{1}, R_{2}, R_{3}$, respectively. Two other symmetric cascaded multilevel inverters have been presented in [13], [14]. These inverters are presented $R_{4}, R_{5}$, respectively. In [15-17], the other cascaded multilevel inverters with symmetric and asymmetric algorithms have been presented. For the presented cascaded multilevel inverter in [15], there are three different algorithms as symmetric and asymmetric states. This inverter with its algorithms are considered $R_{6}, R_{7}, R_{8}$, respectively In this comparison, the presented cascaded multilevel inverters in [16] are considered by $R_{9}$ and $R_{10}$, respectively. The presented inverter in [17] by two different algorithms is indicated $R_{11}, R_{12}$, respectively. Fig. 3 indicates all of cascaded multilevel inverters topologies and Table II shows the presented methods that have been considered for this topologies.

TABLE II. PRESENTED METHODS FOR OTHER STRUCTURES

\begin{tabular}{|c|c|c|}
\hline Topology & Method & $\begin{array}{c}\text { Magnitudes of DC links } \\
\text { for } j=1,2, \ldots, n\end{array}$ \\
\hline \multirow{3}{*}[11]{,$[12]$} & $\mathrm{R} 1$ & $V_{1}=V_{2}=\cdots \quad V_{j} \quad V_{d c}$ \\
\hline & $\mathrm{R} 2$ & $V_{j}=2^{j-1} V_{d c}$ \\
\hline & $\mathrm{R} 3$ & $V_{1}=V_{2}=\cdots \quad V_{j} \quad 2 V_{d c}$ \\
\hline$[13]$ & $\mathrm{R} 4$ & $V_{1}=V_{2}=\cdots \quad V_{j} \quad V_{d c}$ \\
\hline$[14]$ & R5 & $V_{1}=V_{2}=\cdots \quad V_{j} \quad V_{d c}$ \\
\hline \multirow{3}{*}{ [15] } & R6 & $V_{1,1}=V_{2,1}=V_{3,1}=V_{d c} \quad V_{1, j}=\frac{1}{3} V_{2, j}=V_{3, j}=V_{d c}$ \\
\hline & $\mathrm{R} 7$ & $V_{1,1}=V_{2,1}=V_{3,1}=V_{d c}, V_{1, j}=V_{2, j}=V_{3, j}=2 V_{d c}$ \\
\hline & $\mathrm{R} 8$ & $V_{1, j}=V_{2, j}=V_{3, j}=V_{d c}$ \\
\hline \multirow{2}{*}{ [16] } & $\mathrm{R} 9$ & $V_{1,1}=V_{2,1}=V_{d c}, V_{1, j}=V_{2, j}=2 V_{d c}$ \\
\hline & R10 & $V_{1,1}=V_{2,1}=V_{d c}, V_{1, j}=V_{2, j}=3 V_{d c}$ \\
\hline \multirow[t]{2}{*}[17]{} & R11 & $\begin{array}{l}V_{1, n}=\sum_{j=1}^{n-1}\left(V_{1, j}+V_{2, j}\right)+V_{d c}=\frac{3^{n}-1}{2} V_{d c} \\
V_{2, n}=V_{1, n}+V_{d c}=\frac{3^{n}+1}{2} V_{d c} \\
V_{1, j}=V_{2, j}=2 V_{d c}\end{array}$ \\
\hline & $\mathrm{R} 12$ & $V_{1,1}=V_{2,1}=V_{d c}, V_{1, j}=V_{2, j}=3 V_{d c}$ \\
\hline
\end{tabular}

Fig. 4(a) compares the number of IGBTs in the proposed topology with other cascaded multilevel inverters. As it is obvious from this figure, the proposed cascaded inverter needs lower number of IGBTs than other presented inverters in the references except for the presented inverter $R_{12}$. This feature is remarkable when the second proposed algorithm is used. It is pointed out that the bidirectional and unidirectional power switches are used in the proposed cascaded inverter while in the most of topologies only unidirectional power switches are used. In addition, the unidirectional power switches are only used in the presented $R_{12}$. The number of power switches in the proposed cascaded inverter is compared with other inverters.

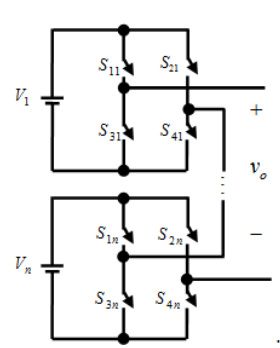

(a)

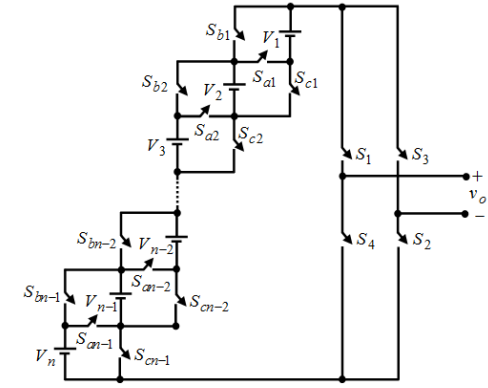

(b)

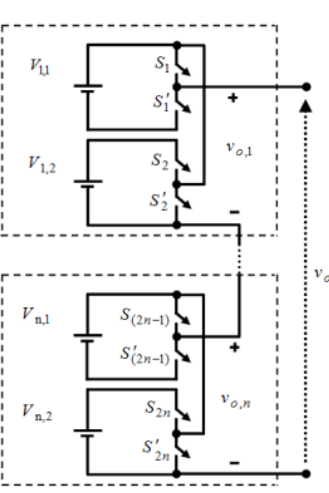

(c)

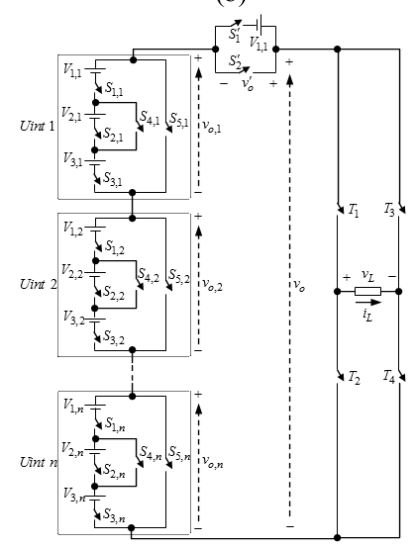

(d)

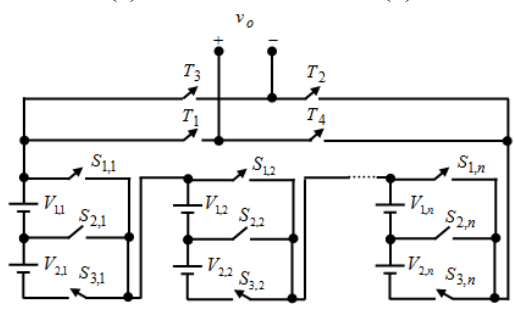

(e)

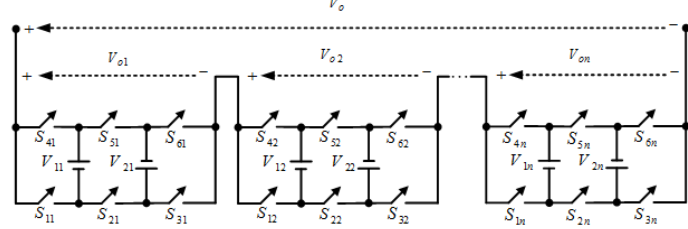

(f)

Fig. 3: The cascaded multilevel inverter structures in literature; (a) $\mathrm{CHB}$; (b) presented in [13]; (c) presented in [14]; (d) presented topology in [15]; (e) presented in [16]; (f) presented in [17] 


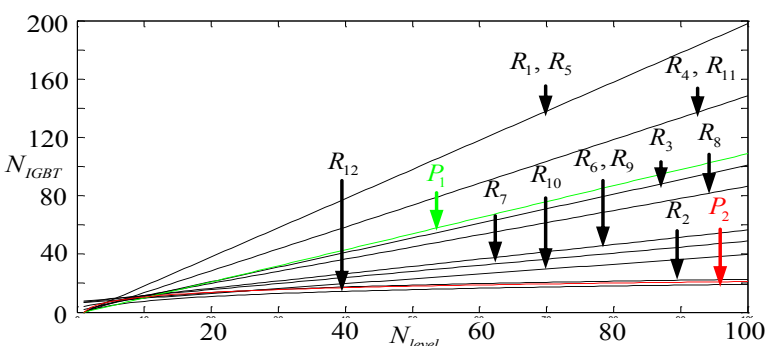

(a)

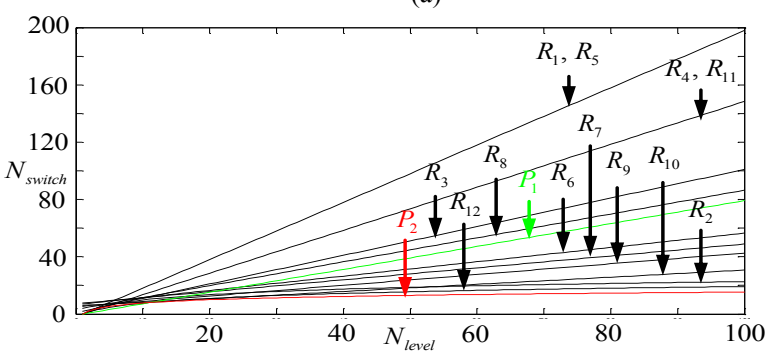

(b)

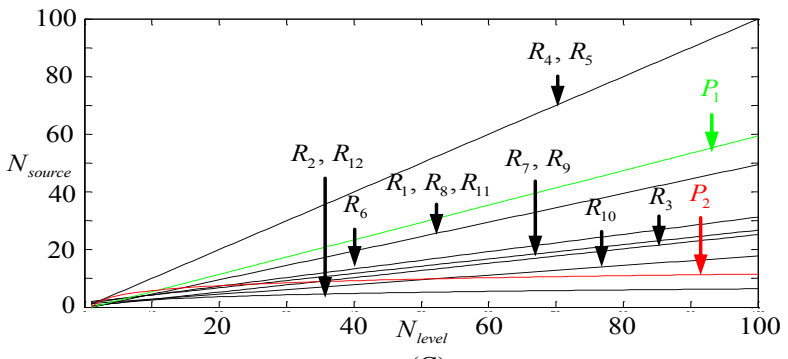

(C)

Fig. 4: Comparison results; (a) Variation of $N_{I G B T}$ versus $N_{\text {level }}$; (b) Variation of $N_{\text {switch }}$ versus $N_{\text {level }}$; (c) Variation of $N_{\text {source }}$ versus $N_{\text {level }}$

This comparison is shown in Fig. 4(b). As it is obvious from this figure, the proposed inverter needs lower number of power switches than other presented inverter in literature. As it is mentioned previously, all unidirectional and bidirectional power switches require a driver circuit. Therefore, the proposed inverter also needs lower number of power diodes than other inverters.

Fig. 4(c) compares the number of dc voltage sources in the proposed topology with other cascaded multilevel inverters. It is clear that the proposed topology based on second proposed algorithm requires lower number of dc voltage sources than other mentioned topologies except the presented inverters $R_{2}, R_{12}$. This is because of the high magnitude of dc voltage sources in the presented inverter as $R_{2}, R_{12}$ that lead to needs lower number of dc voltage sources to generate specific output levels.

It is clear from the comparison; the proposed cascaded inverter is able to generate a higher number of output levels with a lower number of power switches, IGBTs and driver than other topologies. Although the proposed inverter requires a high number of dc voltage source than other topologies but this needs a lower number of power switches and driver circuits to generate specific output level. High number of required dc voltage source can be decreased by using renewable energy sources such as solar cell, wind etc.

\section{EXPERIMENTAL VALIDATIONS}

In this section, the ability of the proposed inverter to generate all voltage levels is reconfirmed by using experimental results on a 49-level inverter. This inverter is shown in Fig. 5. As it is obvious from this figure, this inverter consists of two basic units with ten unidirectional power switches, two bidirectional ones and eight $\mathrm{dc}$ voltage sources. It is important to note that the numbers of power switches in these two basic units are considered equally. In other word $m_{1}=m_{2}=3$. The magnitude of dc voltage sources are determined based on the second proposed algorithm. Based on (22) to (25) and considering $V_{d c}=10 \mathrm{~V}$, the magnitude of dc voltage sources in the first unit is equal to $V_{1}=10 \mathrm{~V}$ and in the second unit is $V_{2}=70 \mathrm{~V}$.

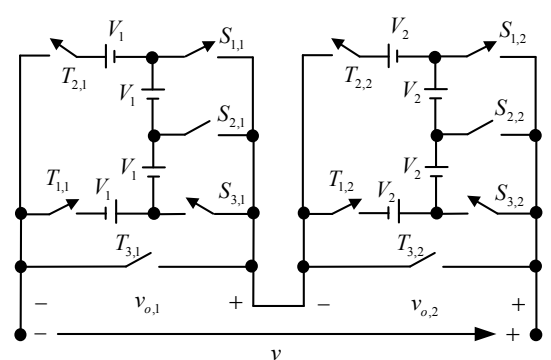

Fig. 5: The proposed 49-level inverter based on the second proposed algorithm

According to (26), this inverter has to be able to generate 49 levels with the maximum amplitude of $240 \mathrm{~V}$ at the output. The IGBTs used on the prototype are HGTP10N40CID (with an internal anti-parallel diode) with the voltage and current ranges of $400 \mathrm{~V}$ and $10 \mathrm{~A}$, respectively. The $89 \mathrm{C} 52$ microcontroller by ATMEL Company has been used to generate of switching patterns. In all process of the experimental performance the load is assumed as $R-L$ load ( $R=107 \Omega, L=55 \mathrm{mH}$ ).

In this paper, the fundamental frequency control method is considered. The main aim of selecting the fundamental frequency control method is its low switching frequency that leads to low switching losses. In this experiment, the switching frequency is $50 \mathrm{~Hz}$.

Figs. 6 show the experimental results. The output voltage waveforms of the first and second basic units are shown in Figs. 6(a) and 6(b), respectively. As shown in Fig. 6(a), the first unit is able to generate positive and negative voltage levels with the amplitudes of $0, \pm 10 \mathrm{~V}, \pm 20 \mathrm{~V}$ and $\pm 30 \mathrm{~V}$. In addition, based on the Fig. 6(b), the second unit is able to generate seven levels with the magnitudes of $0, \pm 70 \mathrm{~V}$, $\pm 140 \mathrm{~V}$ and $\pm 210 \mathrm{~V}$ at the output. The output voltage of this inverter is obtained by adding the generated output levels of the first and second basic units that is shown in Fig. 6(c). According to this figure, the proposed inverter is able to generate 49 levels with the maximum magnitude of $240 \mathrm{~V}$ at the output. The output current of the proposed topology with the maximum amplitude of $2.2 \mathrm{~A}$ is also shown in Fig. 6(c). 


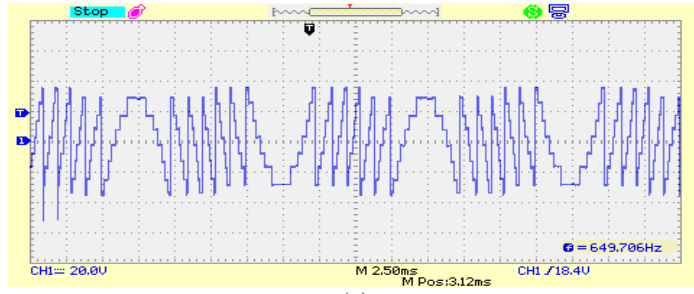

(a)

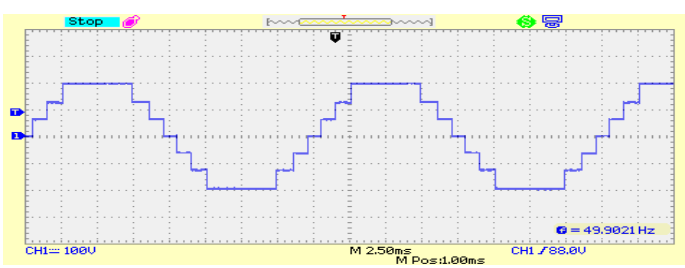

(b)

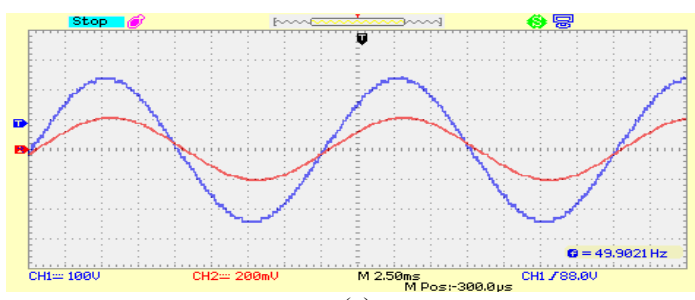

(c)

Fig. 6: Experimental results of the proposed 49-level inverter; (a) the first basic unit output voltage; (b) the second basic unit output voltage; (c) The output voltage and current waveforms.

As mentioned before, the unidirectional and bidirectional power switches from voltage the point of view are used in this topology. As shown in Figs. 7(a) and 7(b) the blocked voltage by switches $S_{1,1}$ is positive and in switch $S_{2,2}$ is there is negative amount on voltage.

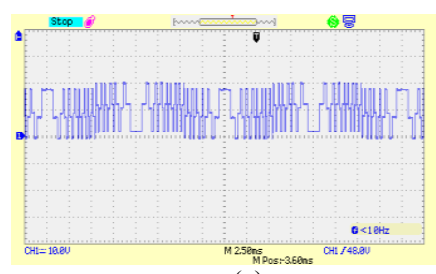

(a)

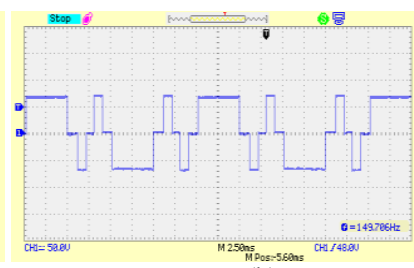

(b)
Fig. 7: The blocked voltage by switches; (a) $S_{1,1}$; (b) $S_{2,2}$.

\section{CONCLUSION}

In this paper, a new basic unit for cascaded multilevel inverters is proposed. By series connection of $n$ number of this basic unit, a new cascaded multilevel inverter is proposed. Then, two different algorithms are proposed to determine the magnitude of dc voltage sources. This inverter requires a lower number of power switches, diodes, driver circuits, and dc voltage sources in comparison with conventional cascaded multilevel inverters that have been presented in the literature. Finally, all of the obtained theoretical issues are reconfirmed by using experimental results on a proposed 49-level inverter based on the second proposed algorithm.

\section{ACKNOWLEDGMENT}

The authors would like to thank the financial support of FONDECYT Regular 1160690 Research Project.

\section{REFERENCES}

[1] K. Maity and T. Roy, "A study of symmetrical and various asymmetrical DC source configurations of a novel cascaded multilevel inverter topology," 2018 Technologies for Smart-City Energy Security and Power (ICSESP), Bhubaneswar, 2018, pp. 1-5.

[2] H. Khoun jahan, K. Zare and M. Abapour, "Verification of a Low Component Nine-Level Cascaded-Transformer Multilevel Inverter in Grid-Tied Mode," IEEE Journal of Emerging and Selected Topics in Power Electronics, vol. 6, no. 1, pp. 429-440, March 2018.

[3] M. M. Hasan, A. Abu-Siada, S. M. Islam and M. S. A. Dahidah, "A New Cascaded Multilevel Inverter Topology with Galvanic Isolation," IEEE Trans. Ind. Appl., vol. 54, no. 4, pp. 3463-3472, July-Aug. 2018.

[4] W. Chen, E. Hotchkiss and A. Bazzi, "Reconfiguration of NPC multilevel inverters to mitigate short circuit faults using back-to-back switches," CPSS Transactions on Power Electronics and Applications, vol. 3, no. 1, pp. 46-55, March 2018.

[5] V. Nair R, K. Gopakumar and L. G. Franquelo, "A Very High Resolution Stacked Multilevel Inverter Topology for Adjustable Speed Drives," IEEE Trans. Ind. Electron., vol. 65, no. 3, pp. 2049-2056, March 2018

[6] S. S. Lee, M. Sidorov, C. S. Lim, N. R. N. Idris and Y. E. Heng, "Hybrid Cascaded Multilevel Inverter (HCMLI) With Improved Symmetrical 4-Level Submodule," IEEE Trans. Power. Electronics, vol. 33, no. 2, pp. 932-935, Feb. 2018.

[7] M. A. Hosseinzadeh, M. Sarbanzadeh, E. Sarbanzadeh, M. Rivera and P. Wheeler, "New Asymmetric Cascaded Multi-level Converter with Reduced Components," IEEE ESARS-ITEC 2018, Nottingham, United Kingdom, 2018, pp. 1-6.

[8] E. Babaei, M. Sarbanzadeh, M. A. Hosseinzadeh and C. Cecati, "A new basic unit for symmetric and asymmetric cascaded multilevel inverter with reduced number of components," IEEE IECON 2016, Florence, 2016, pp. 3147-3152.

[9] N. Susheela, P. S. Kumar and S. K. Sharma, "Generalized Algorithm of Reverse Mapping Based SVPWM Strategy for Diode-Clamped Multilevel Inverters, " IEEE Trans. Ind. Appl., vol. 54, no. 3, pp. 24252437, May-June 2018.

[10] H. Khoun Jahan, M. Abapour and K. Zare, "Switched-Capacitor-Based Single-Source Cascaded H-Bridge Multilevel Inverter Featuring Boosting Ability," IEEE Trans. Power. Electronics, vol. 34, no. 2, pp. 1113-1124, Feb. 2019.

[11] M. Manjrekar and T.A. Lipo, “A hybrid multilevel inverter topology for drive application," in Proc. APEC, 1998, pp. 523-529.

[12] E. Babaei and S.H. Hosseini, "Charge balance control methods for asymmetrical cascaded multilevel converters," in Proc. ICEMS, 2007. Korea, pp. 74-79.

[13] Y. Hinago and H. Koizumi, "A single-phase multilevel inverter using switched series/parallel dc voltage sources," IEEE Trans. Ind. Electron., vol. 57, no. 8, pp. 2643-2650, August 2010.

[14] G. Waltrich and I. Barbi, "Three-phase cascaded multilevel inverter using power cells with two inverter legs in series," IEEE Trans. Ind. Appl., vol. 57, no. 8, pp. 2605-2612, August 2010.

[15] E. Babaei, S. Laali, and Z. Bayat, "A single-phase cascaded multilevel inverter based on a new basic unit with reduced number of power switches," IEEE Trans. Ind. Electron. vol. 62, no. 2, pp. 922-929, Feb. 2015.

[16] S. Laali and E. Babaei, M.B. Bannae Sharifian, "Reduction the number of power electronic devices of a cascaded multilevel inverter based on new general topology," Journal of Operation and Automation in Power Engineering (JOAPE), vol. 2, no. 2, pp. 81-90, Summer/Fall 2014.

[17] M. Sarbanzadeh, E. Babaei, and, S. Laali, "A new basic unit for cascaded multilevel inverters,", in Proc. The 29th (ITC-CSCC), July 14, 2014, Phuket, Thailand, 46-49. 